Discrete Comput Geom 28:671-682 (2002)

DOI: $10.1007 / \mathrm{s} 00454-002-2898-\mathrm{x}$

\title{
A Sufficient Condition for the Existence of Large Empty Convex Polygons*
}

\author{
Pavel Valtr \\ Department of Applied Mathematics, \\ and \\ Institute for Theoretical Computer Science (ITI), Charles University, \\ Malostranské nám. 25, 11800 Praha 1, Czech Republic \\ valtr@kam.mff.cuni.cz
}

\begin{abstract}
Let $P$ be a set of points in general position in the plane. We say that $P$ is $k$-convex if no triangle determined by $P$ contains more than $k$ points of $P$ in the interior. We say that a subset $A$ of $P$ in convex position forms an empty polygon (in $P$ ) if no point of $P \backslash A$ lies in the convex hull of $A$. We show that for any $k, n$ there is an $N=N(k, n)$ such that any $k$-convex set of at least $N$ points in general position in the plane contains an empty $n$-gon. We also prove an analogous statement in $\mathbb{R}^{d}$ for each odd $d \geq 3$.
\end{abstract}

\section{Introduction}

A set $P$ of points in the plane is in general position if it contains no three points on a line. A finite set $X$ of points is in convex position if the points of $X$ are the vertices of a convex polygon.

In 1935 Erdôs and Szekeres proved the following classical result:

Theorem 1 [4]. For any $n \geq 3$, there is an integer $f(n)$ such that any set of at least $f(n)$ points in general position in the plane contains $n$ points in convex position.

We say that a subset $A$ of $P$ in convex position forms an empty polygon if no point of $P \backslash A$ lies in the convex hull of $A$. In 1975 Erdős [3] asked whether the following sharpening of the Erdôs-Szekeres theorem is true: Is there a (smallest) finite $g(n)$ such that any set of at least $g(n)$ points in general position in the plane contains an empty

* This research was supported by project LN00A056 of The Ministry of Education of the Czech Republic, by Charles University Grants Nos. 99/158 and 99/159 and by Czech Republic Grant GAČR 201/99/0242. 
$n$-gon? Somewhat surprisingly, in 1983 Horton [7] gave a negative answer to this question for $n \geq 7$. It is easy to see that $g(3)=3, g(4)=5$. Harborth [6] proved that $g(5)=10$. So far, the existence of $g(6)$ was neither proved nor disproved. In this paper we show that Erdős' question has an affirmative answer if we restrict ourselves to so-called $k$-convex sets.

We say that a finite planar point set $P$ in general position is $k$-convex if no triangle determined by $P$ contains more than $k$ points of $P$ in the interior. Thus, 0 -convex sets are just sets in convex position. Károlyi et al. [8] proved that for any $n$ there is an integer $N(n)$ such that any 1-convex set of at least $N$ points in general position in the plane contains an empty $n$-gon. As far as we know, the 1 -convexity is the first non-trivial condition which was proved to guarantee the existence of an empty $n$-gon in a large set of points in general position. Here we prove that for any fixed $k \geq 1$ the $k$-convexity is also sufficient:

Theorem 2. For any $k, n$ there is an $N=N(k, n)$ such that any $k$-convex set of at least $N$ points in general position in the plane contains an empty $n$-gon.

We conjecture that a statement analogous to Theorem 2 is true also in $\mathbb{R}^{d}$ for any $d \geq 2$. Besides $d=2$, we can prove it for all odd $d \geq 3$ (see Theorem 3 below).

Let $d \geq 2$. We say that a set $P$ of points in $\mathbb{R}^{d}$ is in general position if there are no $\leq d+1$ affinely dependent points in $P$. We say that a finite set of points in $\mathbb{R}^{d}$ is in convex position if none of the points lies in the convex hull of the others. We say that a finite set $P$ of points in general position in $\mathbb{R}^{d}$ is $k$-convex if no simplex determined by $d+1$ points of $P$ contains more than $k$ points of $P$ in the interior.

A notion analogous to empty polygons is the notion of holes. Let $P$ be a set of points in $\mathbb{R}^{d}, d \geq 2$. An $n$-hole of $P$ is any set $X$ of $n$ points of $P$ in convex position such that the convex hull of $X$ contains no other points of $P$.

Theorem 3. For any odd $d \geq 3$ and for any $k, n$, there is an $N=N_{d}(k, n)$ such that any $k$-convex set of at least $N$ points in general position in $\mathbb{R}^{d}$ contains an $n$-hole.

We remark that for any fixed $d \geq 2$ (similarly as for $d=2$ ), if we define $g_{d}(n)$ as the smallest number such that any set of at least $g_{d}(n)$ points in general position in $\mathbb{R}^{d}$ contains an $n$-hole, then $g_{d}(n)$ does not exist for $n$ sufficiently large (see [9]).

Organization of the Paper. The proof of Theorem 2 is given in Section 2. The proof of Theorem 3 is given in Section 3. An alternative proof of Lemma 4 (applied in the proof of Theorem 2) is given in Section 4. Section 5 contains concluding remarks and open problems.

\section{Proof of Theorem 2}

Suppose $k \geq 0$ is fixed. Let $P$ be a $k$-convex set of $N$ points in general position in the plane, where $N$ is very large. 


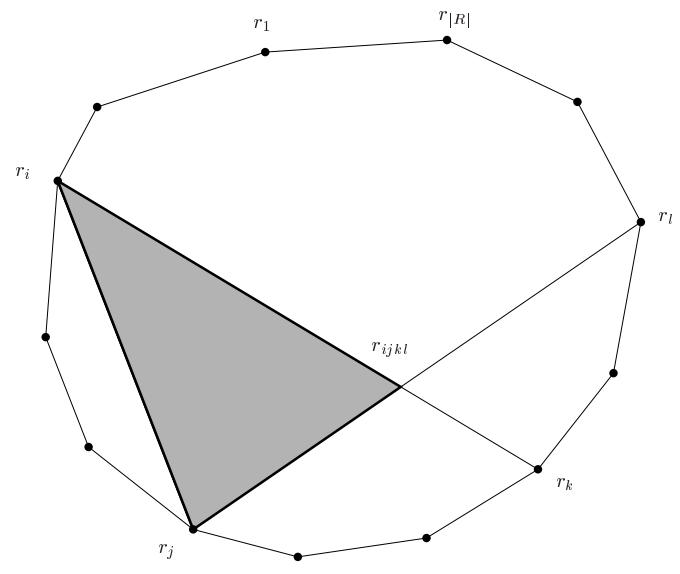

Fig. 1. The triangle $r_{i} r_{j} r_{i j k l}$.

Let $Q \subseteq P$ be the set of vertices of conv $P$. Any triangulation of conv $P=\operatorname{conv} Q$ partitions the interior of conv $P$ into $|Q|-2$ triangles. Since $P$ is $k$-convex, it follows that $|P|=N \leq|Q|+(|Q|-2) k$. Consequently, $|Q|=\Omega(N)$.

We color $\left(\frac{Q}{3}\right)$ by the $k+1$ colors $0,1, \ldots, k$ so that a triple $\left\{q_{1}, q_{2}, q_{3}\right\} \in\left(\frac{Q}{3}\right)$ is colored by color $i$ if the interior of the triangle $q_{1} q_{2} q_{3}$ contains exactly $i$ points of $P$. By Ramsey's theorem, $Q$ contains a monochromatic subset $R$ of size $\Omega(\log \log |Q|)=\Omega(\log \log N)=$ $\Omega\left(\log ^{[2]} N\right)\left(\log ^{[t]}\right.$ denotes $t$ iterations of $\left.\log \right)$. Every triangle determined by $R$ contains a constant number, $c$, of points of $P$ in the interior.

Let $r_{1}, r_{2}, \ldots, r_{|R|}$ be the points of $R$ listed in counterclockwise order. We could start the numbering from any vertex, but from now on the numbering is fixed. Consider the following coloring of $\left(\begin{array}{c}R \\ 4\end{array}\right)$ by $c+1$ colors $0, \ldots, c$. A 4-tuple $\left\{r_{i}, r_{j}, r_{k}, r_{l}\right\}, 1 \leq i<$ $j<k<l \leq|R|$, is colored by color $q$ if the triangle $r_{i} r_{j} r_{i j k l}$ contains exactly $q$ points of $P$ in the interior (where $r_{i j k l}$ denotes the intersection point of the lines $r_{i} r_{k}, r_{j} r_{l}$, see Fig. 1).

Another application of Ramsey's theorem gives a monochromatic subset $S$ of $R$ of size $\Omega\left(\log ^{[3]}|R|\right)=\Omega\left(\log ^{[5]} N\right)$. Let $s_{1}, s_{2}, \ldots, s_{|S|}$ be the points of $S$ listed in the same order as in the sequence $r_{1}, r_{2}, \ldots, r_{|R|}$. Then there are two integers $a, b \geq 0, a+b=c$, such that the following holds. If $s_{i}, s_{j}, s_{k}, s_{l}, 1 \leq i<j<k<l \leq|S|$, are four points of $S$, then the $2 c=2 a+2 b$ points of $P$ inside the quadrilateral $s_{i} s_{j} s_{k} s_{l}$ are partitioned by the diagonals into four subsets of sizes $a, a, b, b$ in the way shown in Fig. 2 (here we use that $S$ is monochromatic and that each triangle determined by $R \supseteq S$ contains exactly $c$ points in the interior).

For $i=2, \ldots,|S|-1$, let $T_{i}$ denote the triangle determined by the lines $s_{i} s_{1}, s_{i} s_{|S|}$, $s_{i-1} s_{i+1}$ (see Fig. 3). Here is the key lemma:

Lemma 4. If $|S| \geq 6$, then $b=0$ and the interior of each of the triangles $T_{i}$ contains exactly $a=c$ points of $P$.

Before proving Lemma 4, we derive Theorem 2 from it. Since conv $S$ can be triangulated into $|S|-2$ triangles, its interior contains exactly $(|S|-2) c$ points of $P$. 


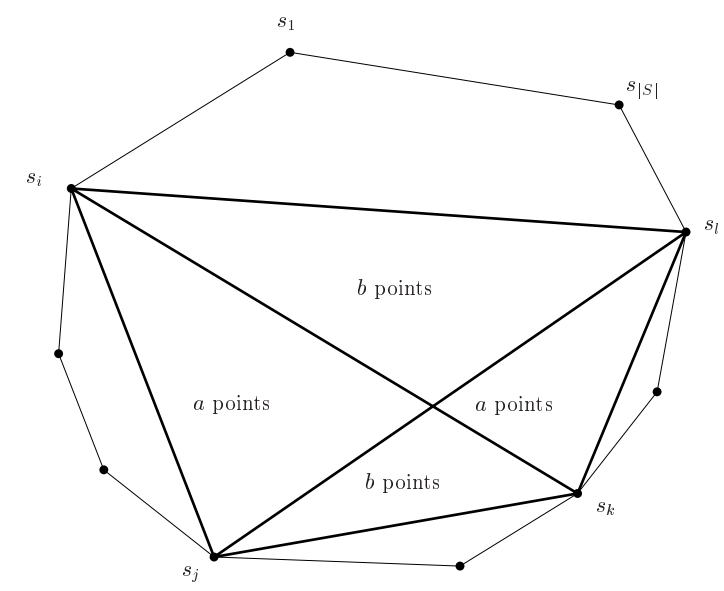

Fig. 2. Distribution of the points in every quadrilateral $s_{i} s_{j} s_{k} s_{l}, 1 \leq i<j<k<l \leq|S|$.

Lemma 4 now shows that conv $S$ contains no point of $P$ outside of $T_{2} \cup T_{3} \cup \cdots \cup T_{|S|-1}$. It follows that if we choose a point $p_{i} \in P$ inside $T_{i}$ closest to the line $s_{i-1} s_{i+1}$, then $s_{1} p_{2} p_{3} \cdots p_{|S|-1} s_{|S|}$ is an empty $|S|$-gon in $P$. Since $|S|=\Omega\left(\log ^{[5]} N\right)$ is at least $n$ for $N$ sufficiently large, Theorem 2 follows. It remains to prove Lemma 4.

We remark that Lemma 4 does not hold for $|S|=5$-Fig. 4 shows a possible placement of points of $P$ inside a pentagon for any $a \geq b \geq 0, a+b=c$.

Proof of Lemma 4 . We first prove that $b=0$. Figure 5 shows the two non-degenerate cases differing by the way the diagonals partition the interior of the hexagon $s_{1} s_{2} s_{3} s_{4} s_{5} s_{6}$ (the remaining degenerate case when the diagonals $s_{1} s_{4}, s_{2} s_{5}, s_{3} s_{6}$ have a common intersection point can be seen as a special case of any of them). We treat the two cases at the same time - they are very similar.

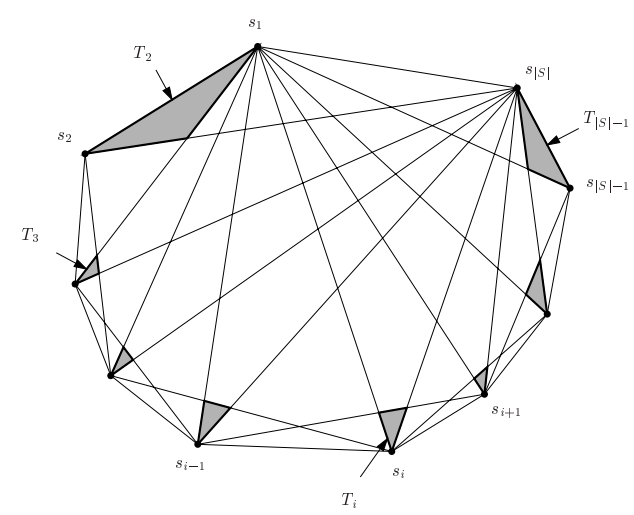

Fig. 3. The triangles $T_{i}$. 


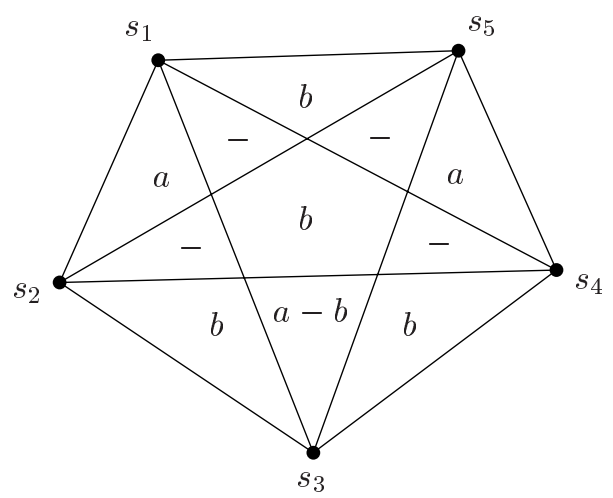

Fig. 4. Possible distribution of points inside the pentagon $s_{1} s_{2} s_{3} s_{4} s_{5}$, if $|S|=5, a \geq b \geq 0, a+b=c$.

For each picture in Fig. 5, consider the regions $R_{1}$ through $R_{12}$ (or $R_{13}$ ). For a region $X$, let $|X|$ denote the number of points of $P$ lying in the interior of $X$.

We have $\left|R_{1}\right|=a$ (due to the partition of the points in the quadrilateral $s_{1} s_{2} s_{3} s_{6}$ ) and $\left|R_{4}\right|=b$ (quadrilateral $s_{1} s_{2} s_{5} s_{6}$ ). Consequently, we get $\left|R_{3}\right|=\left|R_{5}\right|=\left|R_{10}\right|=0$ from $\left|R_{3}\right|+\left|R_{4}\right|+\left|R_{5}\right|+\left|R_{10}\right| \leq b$ (quadrilateral $\left.s_{1} s_{3} s_{4} s_{6}\right)$ and also $\left(\left|R_{13}\right|=\right)\left|R_{2}\right|=\left|R_{8}\right|=$ $\left|R_{9}\right|=0$ from $\left(\left|R_{13}\right|+\right)\left|R_{1}\right|+\left|R_{2}\right|+\left|R_{8}\right|+\left|R_{9}\right|=a$ (quadrilateral $s_{1} s_{2} s_{4} s_{5}$ ).

Analogously, $\left|R_{7}\right|=a$ and thus $\left|R_{6}\right|=\left|R_{11}\right|=\left|R_{12}\right|=0$ follows from $\left(\left|R_{13}\right|+\right)$ $\left|R_{6}\right|+\left|R_{7}\right|+\left|R_{11}\right|+\left|R_{12}\right|=a$. Thus, $\left(\left|R_{13}\right|+\right)\left|R_{8}\right|+\left|R_{9}\right|+\left|R_{10}\right|+\left|R_{11}\right|+\left|R_{12}\right|=0$ but this quantity also equals $b$ (quadrilateral $s_{1} s_{2} s_{5} s_{6}$ ). Thus, $b=0$ (and $a=c$ ).

We now easily show that $\left|T_{i}\right|=a$ for each $i$. We have $\left|T_{2}\right|=a$ (quadrilateral $s_{1} s_{2} s_{3} S_{|S|}$ ) and $\left|T_{|S|-1}\right|=a$ (quadrilateral $s_{1} s_{|S|-2} s_{|S|-1} s_{|S|}$ ). For $i=3, \ldots,|S|-2$, let $U_{i}$ denote the triangle $s_{i-1} s_{i} x_{i}$, where $x_{i}$ is the intersection point of the diagonals $s_{1} s_{i}$, $s_{i-1} s_{i+1}$ (see Fig. 6). Then $\left|U_{i}\right|=b=0$ (quadrilateral $s_{1} s_{i-1} s_{i} s_{i+1}$ ) and $\left|U_{i} \cup T_{i}\right|=a$ (quadrilateral $s_{i-1} s_{i} s_{i+1} s_{|S|}$ ). Thus, $\left|T_{i}\right|=a$ for each $i$.
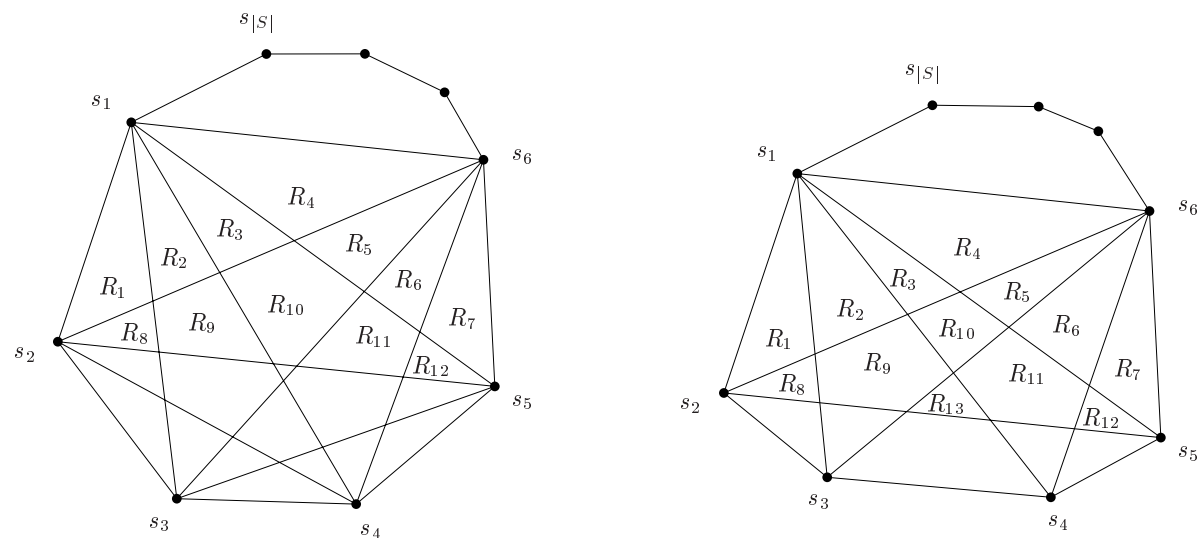

Fig. 5. Possible partitions of the interior of the hexagon $s_{1} s_{2} s_{3} s_{4} s_{5} s_{6}$ and the regions $R_{i}$. 


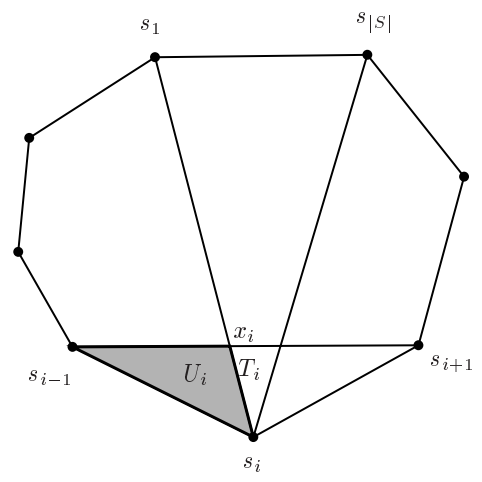

Fig. 6. The region $U_{i}$.

\section{Proof of Theorem 3}

Let $d \geq 3$ be odd. We may suppose that $n \geq d+2$. Let $P$ be a $k$-convex set of $N$ points in general position in $\mathbb{R}^{d}$, where $N=N_{d}(k, n)$ is very large. By a generalization of the Erdős-Szekeres theorem to higher dimensions (e.g., see Exercise 7.3.6 of [5] or Corollary 3.8 of [2]), $P$ contains a subset $Q$ of $N^{\prime}$ vertices of a cyclic polytope in $\mathbb{R}^{d}$, where $N^{\prime}=N_{d}^{\prime}(k, n)$ is still large. Since $P$ is $k$-convex, we can color $\left(\begin{array}{c}Q \\ d+1\end{array}\right)$ by the $k+1$ colors $0,1, \ldots, k$ so that a $(d+1)$-tuple $\left\{q_{1}, \ldots, q_{d+1}\right\} \in\left(\begin{array}{c}Q \\ d+1\end{array}\right)$ is colored by color $i$ if the interior of the simplex with the vertex set $\left\{q_{1}, \ldots, q_{d+1}\right\}$ contains exactly $i$ points of $P$. If $N^{\prime}$ is sufficiently large, then, by Ramsey's theorem, $Q$ contains a monochromatic subset $R$ of size $n$. Every simplex determined by $R$ contains a constant number, $c$, of points in the interior. We will show that $c=0$.

Let $r_{1}, \ldots, r_{n}$ be the points of $R$ listed in the "cyclic-polytope" order. Set

$$
\begin{aligned}
C & :=\operatorname{conv}\left\{r_{1}, \ldots, r_{d+2}\right\}, \\
C_{i} & :=\operatorname{conv}\left\{r_{1}, \ldots, r_{i-1}, r_{i+1}, \ldots, r_{d+2}\right\}, \quad \text { for } i=1, \ldots, d+2 .
\end{aligned}
$$

Lemma 5. The cyclic polytope $C$ can be triangulated into simplices in the following two ways: $C=C_{1} \cup C_{3} \cup \cdots \cup C_{d+2}$ and $C=C_{2} \cup C_{4} \cup \cdots \cup C_{d+1}$.

Proof. If $i \neq j$ are of the same parity, then the interiors of $C_{i}, C_{j}$ are disjoint because $C_{i}, C_{j}$ lie on different sides of their common facet $\operatorname{conv}\left\{r_{\alpha}: 1 \leq \alpha \leq d+2, \alpha \neq i, j\right\}$. It remains to show that $C \subseteq C_{1} \cup C_{3} \cup \cdots \cup C_{d+2}$ and $C \subseteq C_{2} \cup C_{4} \cup \cdots \cup C_{d+1}$.

The unique Radon partition of the set $R^{\prime}:=\left\{r_{1}, \ldots, r_{d+2}\right\}$ is $R^{\prime}=R_{\text {odd }}^{\prime} \cup R_{\text {even }}^{\prime}$, where $R_{\text {odd }}^{\prime}=\left\{r_{1}, r_{3}, \ldots, r_{d+2}\right\}, R_{\text {even }}^{\prime}=\left\{r_{2}, r_{4}, \ldots, r_{d+1}\right\}$ (this is well known and follows from the fact that any other pair of disjoint subsets of $R^{\prime}$ is separated by a hyperplane). Algebraically,

$$
\begin{aligned}
\sum_{i \text { odd }} \beta_{i} r_{i}= & \sum_{i \text { even }} \beta_{i} r_{i}, \\
\text { where } & \sum_{i \text { odd }} \beta_{i}=\sum_{i \text { even }} \beta_{i}=1, \quad \beta_{i} \geq 0 \quad(i=1, \ldots, d+2) .
\end{aligned}
$$


Let $x \in C$. Then

$$
x=\sum_{i=1}^{d+2} \alpha_{i} r_{i}, \quad \text { where } \quad \sum_{i=1}^{d+2} \alpha_{i}=1, \quad \alpha_{i} \geq 0 \quad(i=1, \ldots, d+2) .
$$

If we sum up the equality $x=\sum_{i=1}^{d+2} \alpha_{i} r_{i}$ and a proper non-negative multiple of the equality

$$
0=\sum_{i \text { even }} \beta_{i} r_{i}-\sum_{i \text { odd }} \beta_{i} r_{i}
$$

we obtain an equality

$$
x=\sum_{i=1}^{d+2} \alpha_{i}^{\prime} r_{i}, \quad \text { where } \quad \sum_{i=1}^{d+2} \alpha_{i}^{\prime}=1, \quad \alpha_{i}^{\prime} \geq 0 \quad(i=1, \ldots, d+2),
$$

such that $\alpha_{i}^{\prime}=0$ holds for at least one odd index $i$. It follows that $x \in C_{i}$ and thus $C \subseteq C_{1} \cup C_{3} \cup \cdots \cup C_{d+2}$. Similarly we can prove that $C \subseteq C_{2} \cup C_{4} \cup \cdots \cup C_{d+1}$.

Since the interior of each of the simplices $C_{i}$ contains the same number, $c$, of points of $P$, Lemma 5 gives $((d+3) / 2) c=((d+1) / 2) c$ and thus $c=0$. Thus, the interior of the convex hull of $R$ contains no point of $P$. This concludes the proof of Theorem 3 .

We remark that if $d$ is even, then every triangulation of a cyclic polytope in $\mathbb{R}^{d}$ partitions the polytope into the same number, $\left(\begin{array}{c}n-d / 2-1 \\ d / 2\end{array}\right)$, of simplices (because any two triangulations in this situation are connected by a finite number of flips that preserve the triangulation size). Therefore the above proof does not work if $d$ is even.

\section{Second Proof of Lemma 4}

Here we give an alternative proof of Lemma 4. We believe that this proof (rather than the proof given in Section 2) might have a generalization in even dimensions which would consequently give an analogue of Theorems 2 and 3 in even dimensions.

We may suppose that $|S|=2 m$ is even. For $i=1,2, \ldots, 2 m-1$, let $v_{i}$ be the point of intersection of the main diagonals $s_{i} s_{i \pm m}, s_{i+1} s_{i+1 \pm m}$ (we always choose "+" or "-" so that the obtained index lies in the range $\{1,2, \ldots, 2 m\})$ and let $V_{i}$ be the triangle $s_{i} s_{i+1} v_{i}$. Further, put $v_{2 m}:=v_{m}$ and let $V_{2 m}$ be the triangle $s_{2 m} s_{1} v_{2 m}$.

We first show that the triangles $V_{1}, \ldots, V_{2 m}$ cover conv $S$. This is clearly true for $m=2$ (we have $v_{1}=v_{2}=v_{3}=v_{4}$ in this case). We further proceed by induction on $m$. Let $m \geq 3$. We denote by $x$ the point of intersection of the diagonals $s_{1} s_{m+1}, s_{3} s_{m+3}$ (see Fig. 7). By the induction assumption, $\operatorname{conv}\left(S \backslash\left\{s_{2}, s_{m+2}\right\}\right)$ is covered by the $2 m-2$ triangles $\Delta s_{1} s_{3} x, V_{3}, V_{4}, \ldots, V_{m}, \Delta s_{m+1} s_{m+3} x, V_{m+3}, V_{m+4}, \ldots, V_{2 m}$. The statement now follows from the following three facts (see Fig. 7): (a) conv $S=$ $\operatorname{conv}\left(S \backslash\left\{s_{2}, s_{m+2}\right\}\right) \cup \triangle s_{1} s_{2} s_{3} \cup \triangle s_{m+1} s_{m+2} s_{m+3}$, (b) $\triangle s_{1} s_{3} x \cup \triangle s_{1} s_{2} s_{3}$ is covered by $V_{1} \cup V_{2}$, (c) $\triangle s_{m+1} s_{m+3} x \cup \Delta s_{m+1} s_{m+2} s_{m+3}$ is covered by $V_{m+1} \cup V_{m+2}$.

For each triangle $V_{i}$, we now find a smaller triangle $V_{i}^{\prime}$ contained in $V_{i}$ such that the interiors of the triangles $V_{i}, V_{i}^{\prime}$ contain the same number of points of $P$. Then $V_{i} \backslash V_{i}^{\prime}$ will 

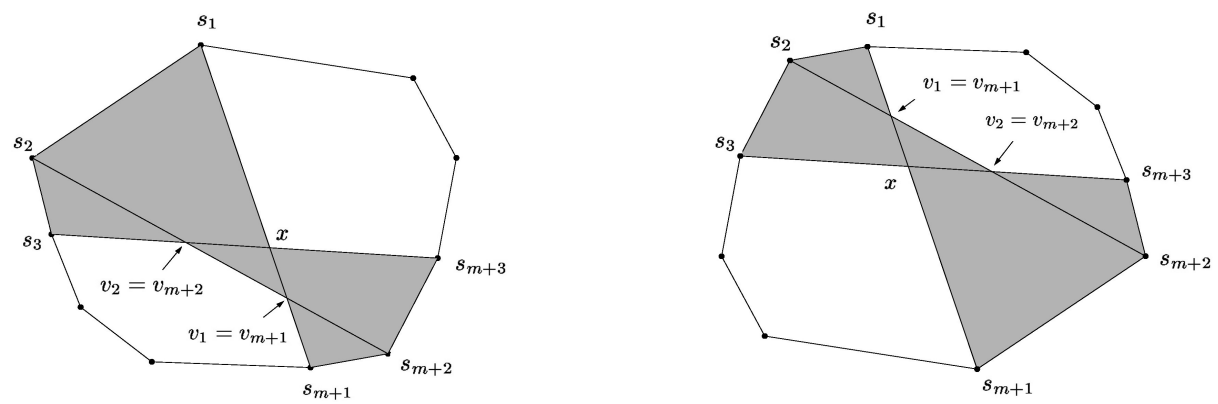

Fig. 7. The point $x$ and the main diagonals $s_{i} s_{m+i}, i=1,2,3$ (two combinatorially different cases). The shaded region is covered by $V_{1} \cup V_{2} \cup V_{m+1} \cup V_{m+2}$.

contain no point of $P$. Since conv $S=V_{1} \cup \cdots \cup V_{2 m}$, points of $P$ inside conv $S$ will lie in $V_{1}^{\prime} \cup \cdots \cup V_{2 m}^{\prime}$.

For $i=1, \ldots, m-1$, we define $V_{i}^{\prime}$ as the intersection of the triangles $s_{i} s_{i+1} s_{2 m}$ and $s_{i} s_{i+1} s_{i+2}$. For $i=m+1, \ldots, 2 m-1$, we define $V_{i}^{\prime}$ as the intersection of the triangles $s_{1} s_{i} s_{i+1}$ and $s_{i-1} s_{i} s_{i+1}$. Further, we define $V_{m}^{\prime}$ as the intersection of the triangles $s_{m-1} s_{m} s_{m+1}$ and $s_{m} s_{m+1} s_{m+2}$, and we define $V_{2 m}^{\prime}$ as the intersection of the triangles $s_{2 m} s_{1} s_{2}$ and $s_{2 m-1} s_{2 m} s_{1}$ (see Fig. 8).

Clearly, if $i \in\{1, \ldots, 2 m\}$, then $V_{i}^{\prime} \subset V_{i}$ and, by the choice of $S$, the interiors of $V_{i}^{\prime}, V_{i}$ contain the same number ( $a$ or $b$ ) of points of $P$. Thus, as argued above, all points of $P$ inside conv $S$ lie in $V_{1}^{\prime} \cup \cdots \cup V_{2 m}^{\prime}$. By the choice of $S$, the interiors of $V_{m}^{\prime}$ and $V_{2 m}^{\prime}$ contain $b$ points each, and the interior of any other triangle $V_{i}^{\prime}$ contains $a$ points of $P$. Since the triangle $s_{1} s_{2} s_{2 m-1}$ contains $V_{1}^{\prime}$ and is disjoint from the interiors of all other triangles $V_{i}^{\prime}$, its interior contains $a$ points of $P$. At the same time, it contains $c$ points of

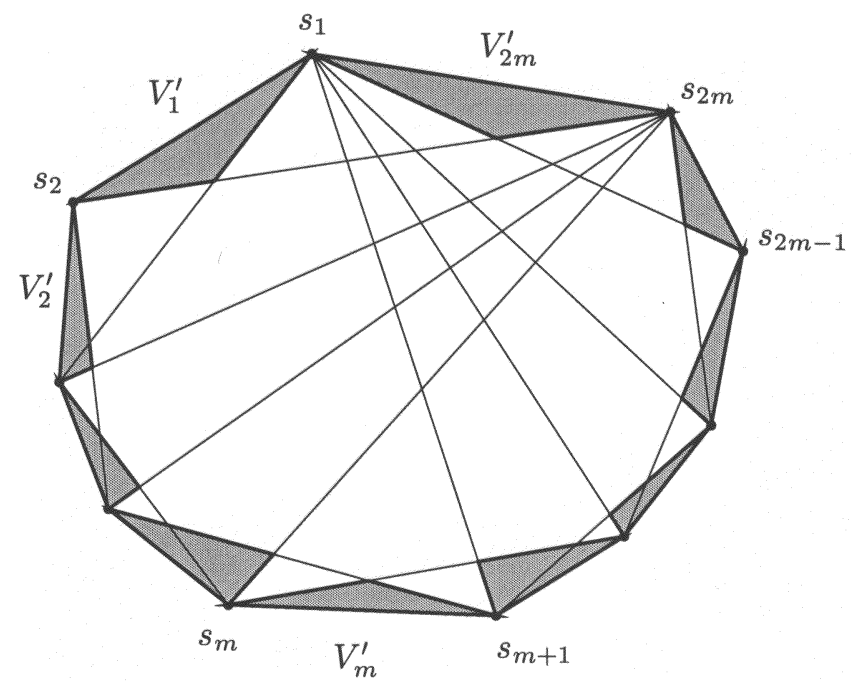

Fig. 8. The triangles $V_{i}^{\prime}$. 
$P$. It follows that $a=c$ and thus $b=0$. The interior of $V_{2 m}^{\prime}$ contains $b=0$ points of $P$. Therefore, each triangle $s_{1} s_{i} s_{2 m}, i=2, \ldots, 2 m-1$, contains its $a=c$ interior points belonging to $P$ in its intersection with $V_{i}^{\prime}$, which is the triangle $T_{i}$.

\section{Concluding Remarks and Open Problems}

\subsection{The Modular Version of the Erdös-Szekeres Theorem}

Károlyi et al. [8] studied 1-convex sets in relation to the following conjecture:

Conjecture 1 [1]. For any $n \geq 3$ and $p \geq 2$, there exists an integer $B=B(n, p)$ such that every set $P$ of at least $B$ points in general position in the plane determines a convex $n$-gon such that the number of points of $P$ in its interior is divisible by $p$.

Conjecture 1 was proved in [1] for every $n \geq p+2$, Károlyi et al. [8] proved it for every $n \geq 5 p / 6+O(1)$. In their proof they applied the special case $k=1$ of Theorem 2 . In fact, Theorem 2 gives Conjecture 1 for all $n \geq 3 p / 4+O(1)$. This is shown in [10]. In [10] we also discuss a $d$-dimensional version of Conjecture 1 and prove it if $d \geq 3$ is odd or $n \geq n_{0}(p, d)$.

\subsection{A Cap or an (Empty) Cup}

We believe that point sets with no large holes have a very special structure. Therefore, it is interesting to seek conditions ensuring the existence of a hole of any fixed size in every sufficiently large point set in $\mathbb{R}^{d}$. One such condition ( $k$-convexity) is given in Theorems 2 and 3.

We say that a set $X$ of $n$ points in the plane, no two on a vertical line, is an $n$-cap ( $n$-cup, respectively) if it is in convex position and all points of $X$ lie on and above (on and below, respectively) the line connecting the leftmost point of $X$ with the rightmost point of $X$ (see Fig. 9).

In their classical paper [4], Erdős and Szekeres proved that any set of at least $\left(\begin{array}{c}2 n-4 \\ n-2\end{array}\right)+1$ points in general position in the plane, no two of them on a vertical line, contains an $n$-cap or an $n$-cup. From this they concluded that Theorem 1 holds with $f(n):=\left(\begin{array}{c}2 n-4 \\ n-2\end{array}\right)+1$.

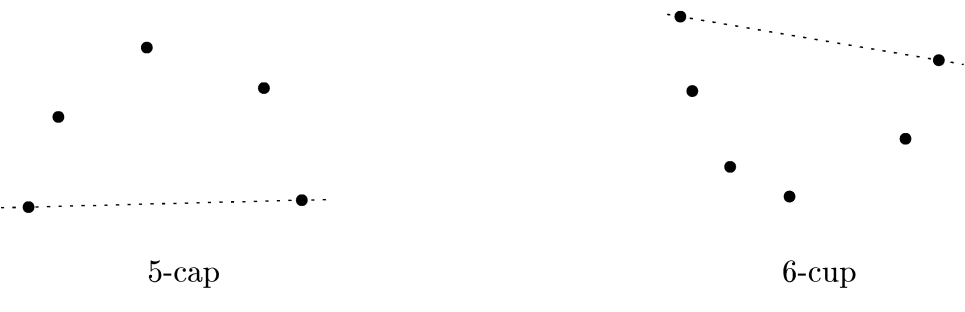

Fig. 9. A cap and a cup. 
We conjecture that actually the following stronger result holds:

Conjecture 2. For any $n \geq 3$, there is an integer $f^{\prime}(n)$ such that any set of at least $f^{\prime}(n)$ points in general position in the plane, no two of them on a vertical line, contains an $n$-cap or an empty $n$-cup. (An empty $n$-cup is an $n$-cup which forms an empty $n$-gon.)

Conjecture 2, if true, would imply that for any $k$ and $n$ any sufficiently large set of points in the plane with no $k$-cap contains an empty $n$-gon.

\subsection{Strengthenings of Theorems 2 and 3}

Theorems 2 and 3 can be generalized in the following two ways:

- the general position is not necessary,

- the statement is true also if the considered set is a union of a fixed number of $k$-convex sets.

These generalizations may be combined in one theorem:

Theorem 6. If $d=2$ or $d \geq 3$ is odd, then, for any three positive integers $k, l, n$, there is an $N=N(d, k, l, n)$ with the following property. Let $P=P_{1} \cup P_{2} \cup \cdots \cup P_{l}$ be a set of $N$ points in $\mathbb{R}^{d}$ such that for each $i$ the convex hull of any $d+1$ points of $P_{i}$ contains at most $k$ other points of $P_{i}$. Then the set $P$ has an $n$-hole.

Proof. Suppose that $d=2$, that $k, l, n$ are three positive integers, and that $N$ is very large. Let $P=P_{1} \cup P_{2} \cup \cdots \cup P_{l}$ be as in the theorem. Without loss of generality, we may suppose that the sets $P_{i}$ are pairwise disjoint and that $\left|P_{1}\right| \geq N / l$. The set $P_{1}$ cannot contain more than $k+3$ points on a line (otherwise the convex hull of three of them would contain more than $k$ other points of $P_{1}$ ). A greedy algorithm gives a set $P_{1}^{\prime}$ of $N_{1}$ points of $P_{1}$ in general position such that no segment determined by two points of $P_{1}^{\prime}$ contains other points of $P_{1}$ and $N_{1}$ is still very large. By the same proof as for Theorem 2, we can show that $P_{1}^{\prime}$ has a subset $P_{1}^{\prime \prime}$ forming an empty $N_{1}^{\prime}$-gon in $P_{1}$, where $N_{1}^{\prime}$ is still very large. If conv $P_{1}^{\prime \prime}$ contains fewer than $N_{1}^{\prime} / n$ of points of $P_{2} \cup \cdots \cup P_{l}$, then $P_{1}^{\prime \prime}$ contains $n$ points forming an empty $n$-gon in $P_{1} \cup \cdots \cup P_{l}$. Otherwise, we consider the points of $P \backslash P_{1}$ lying in conv $P_{1}^{\prime \prime}$. Their number is at least $N_{1}^{\prime} / n$, which is still a very large number. If we repeat the same argument (at most) $l-1$ times, we obtain an empty $n$-gon in $P_{1} \cup \cdots \cup P_{l}$ formed by $n$ points of one of the sets $P_{2}, \ldots, P_{l}$.

If $d \geq 3$ is odd, we can proceed similarly, applying Theorem 3 instead of Theorem 2 .

\subsection{A Density Statement Does Not Hold}

It does not suffice that $99 \%$ of the simplices determined by $P$ are empty:

Claim 7. For any $d \geq 2$ and for any $\varepsilon>0$, there is an integer $c=c(d, \varepsilon) \geq 3$ such that for any $N \geq d+1$ there is a set $P$ of $N$ points in general position in $\mathbb{R}^{d}$ such 


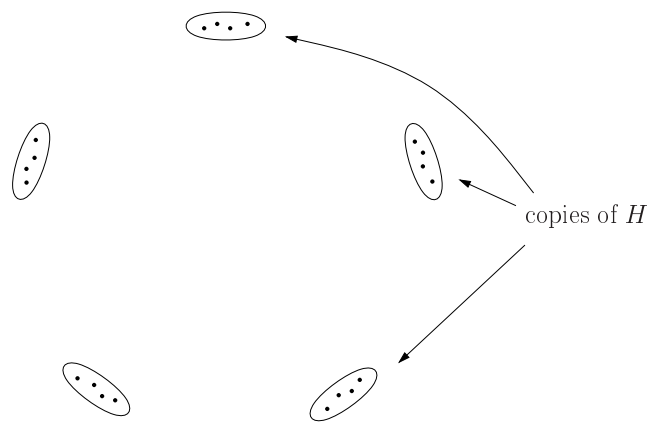

Fig. 10. $3 / \varepsilon$ copies of $H$ along a circle.

that more than $(1-\varepsilon)\left(\begin{array}{c}|P| \\ d+1\end{array}\right)$ simplices determined by $P$ are $(d+1)$-holes $($ i.e., empty simplices), but the set $P$ does not contain a c-hole.

Proof. First, let $d=2$. Suppose for simplicity that $3 / \varepsilon$ is an integer and that $N$ is divisible by $3 / \varepsilon$. Let $H$ be a set of $N /(3 / \varepsilon)=\varepsilon N / 3$ points in general position in the plane with no empty 7-gon (arbitrarily large sets with no empty 7-gon were constructed by Horton [7]). We shrink the second coordinates of points in $H$ (without changing the combinatorial structure of $H$ ) so that all lines determined by $H$ will be almost horizontal. Further, we take $3 / \varepsilon$ copies of $H$ rotated along a large circle (see Fig. 10). The union of these copies will be our set $P$. It contains no empty convex $(18 / \varepsilon+1)$-gon (an empty polygon cannot contain seven points from a copy of $H$ ). Moreover, each triple of points from distinct copies of $H$ is empty. There are more than

$$
\frac{3 / \varepsilon-1}{3 / \varepsilon} \cdot \frac{3 / \varepsilon-2}{3 / \varepsilon}\left(\begin{array}{l}
n \\
3
\end{array}\right)>(1-\varepsilon)\left(\begin{array}{l}
n \\
3
\end{array}\right)
$$

such triples. This completes the case $d=2$. If $d>2$, we proceed similarly, using the construction of [9] of arbitrarily large sets of points in general position in $\mathbb{R}^{d}$ with no $c_{d}$-hole, where $c_{d}$ is a constant depending on $d$ only.

\section{Acknowledgment}

The author is indebted to Gyula Károlyi for interesting and motivating discussions. Alex Below and Jesus De Loera explained some properties of the cyclic polytope triangulations. The referees are also thanked for their helpful suggestions.

\section{References}

1. A. Bialostocki, P. Dierker, and B. Voxman, Some notes on the Erdős-Szekeres theorem, Discrete Math. 91 (1991), 231-238.

2. R. Cordovil and P. Duchet, Cyclic polytopes and oriented matroids, European J. Combin. 21 (2000), 49-64. 
3. P. Erdős, On some problems of elementary and combinatorial geometry, Ann. Mat. Pura Appl. (4) 103 (1975), 99-108.

4. P. Erdős and Gy. Szekeres, A combinatorial problem in geometry, Compositio Math. 2 (1935), 464-470.

5. B. Grünbaum, Convex Polytopes, Pure and Applied Mathematics, Vol. 16, Wiley, New York, 1967.

6. H. Harborth, Konvexe Fünfecke in ebenen Punktmengen, Elem. Math. 33 (1978), 116-118.

7. J. D. Horton, Sets with no empty convex 7-gons, Canad. Math. Bull. 26 (1983), 482-484.

8. Gy. Károlyi, J. Pach, and G. Tóth, A modular version of the Erdős-Szekeres theorem, Studia Sci. Math. Hungar. 38 (2001), 245-259.

9. P. Valtr, Sets in $\mathbb{R}^{d}$ with no large empty convex subsets, Discrete Math. 108 (1992), 115-124.

10. P. Valtr, On the modular version of the Erdős-Szekeres theorem, in preparation.

Received April 18, 2001, and in revised form March 21, 2002. Online publication October 29, 2002.

Note added in proof. An alternative proof of Theorem 2 is given in the very recent manuscript by G. Kun and G. Lippner, "Large convex empty polygons in $k$-convex sets". The proof gives $N(k, n) \leq 2^{(k+2)^{n+1}}$. 